\title{
Percutaneous occlusion of large aneurysmal right coronary artery saphenous vein bypass graft
}

\author{
Wiktor Kuliczkowski ${ }^{1}$, Jacek Kusa², Piotr Brzozowski ${ }^{3}$, Agnieszka Witkowska', Luiza Bulak ${ }^{3}$, Krzysztof Reczuch $^{1}$ \\ ${ }^{1}$ Institute of Heart Diseases, Wroclaw Medical University, Wroclaw, Poland \\ 2Pediatric Cardiology Department, Regional Specialist Hospital, Wroclaw, Poland \\ ${ }^{3}$ University Teaching Hospital, Wroclaw, Poland
}

Adv Interv Cardiol 2021; 17, 3 (65): 313-314

DOI: https://doi.org/10.5114/aic.2021.109237

An 80-year-old man after a coronary artery bypass graft (CABG) in 2003 (LIMA-LAD, SVG-RCA, SVG-Dg) had a chest $X$-ray scan performed due to pulmonary infection which uncovered a mediastinal tumor. Using computed tomography SVG-RCA aneurysm consisting of two communicationg sacs $(8.2 \times 8.6 \times 8.4 \mathrm{~cm}$ and $9.8 \times 8.1 \times 9.2 \mathrm{~cm})$ were detected (Figures $1 \mathrm{~A}, \mathrm{~B}$ ). The patient did not show any symptoms related to the aneurysm. The patient was consulted by the Heart Team in accordance with the literature regarding the risk of further enlargement or rupture of the aneurysms [1], and due to high risk of complications during sternotomy in the cardiosurgeons' assessment the decision was taken to close the aneurysms percutaneously. The bypass graft was intubated from the right femoral artery using an $8 \mathrm{~F}$ Cook Flexor guiding catheter. Before the occlusion, an $8 \mathrm{~mm}$ diameter balloon was inflated for $15 \mathrm{~min}$ in the proximal part of the graft, resulting in only a transient elevation of ST segments in II, III and aVF leads lasting for about $5 \mathrm{~min}$ with no typical pain. In coronary angiography, the distal part of the RCA was hardly supplied from the aneurysmal graft, but good and above mentioned control collaterals were visible from the left coronary artery, and above mentioned control balloon inflation was performed to check their efficiency. Due to diffused atherosclerotic lesions in the proximal and medial parts of the RCA, revascularization was not attempted. As we only observed transient ST segment elevation during the balloon inflation a "Vascular Plug II" (Abbott) size $10 \mathrm{~mm}$ vascular occluder was implanted in the proximal part of the graft (Figure $1 \mathrm{C}$ ). Total occlusion of the graft's ostium was accomplished with no contrast flowing into the lumen of the aneurysm in follow-up angiography (Figure $1 \mathrm{D}$ ). Occlusion of the distal part of the aneurysm was technically difficult and was not performed. Despite collaterals of the RCA from the left coronary artery, elevated levels of troponin were noted in the periprocedural period with inferior segments' hypokinesis. The patient was discharged in good overall condition 8 days after the procedure. Sadly, no follow-up information is available, due to the patient's death caused by SARS- CoV2 infection.

Saphenous vein graft aneurysms are a rare condition estimated by some authors to be present in $0.07 \%$ of CABG patients. The incidence rate may be higher given the frequent asymptomatic occurrence of especially smaller aneurysms [1]. Aneurysms bigger than $10 \mathrm{~cm}$ in diameter are considered to be at higher risk of rupture, and it is believed that they should be treated even if asymptomatic [1, 2]. Treatment options include surgery or percutaneous techniques such as implantation of various vascular plugs, covered stents, and arterial coiling [1, 2].

We report two giant compartment aneurysms of the vein graft to the RCA which were closed percutaneously. The decision to close the whole graft with compromises to the blood flow to the otherwise amputated native artery was preceded by transient graft occlusion with a balloon to check for collaterals in efficiency from the left coronary artery. Despite a good result of the test and reassuring data from the literature [3] the patient developed periprocedural myocardial infarction, which points to an uncertain outcome and individualized approach in this rare condition.

\section{Conflict of interest}

The authors declare no conflict of interest.

Corresponding author:

Piotr Brzozowski, University Teaching Hospital, Wroclaw, Poland, e-mail: p.brzozowski95@gmail.com

Received: 19.05.2021, accepted: 23.08.2021. 

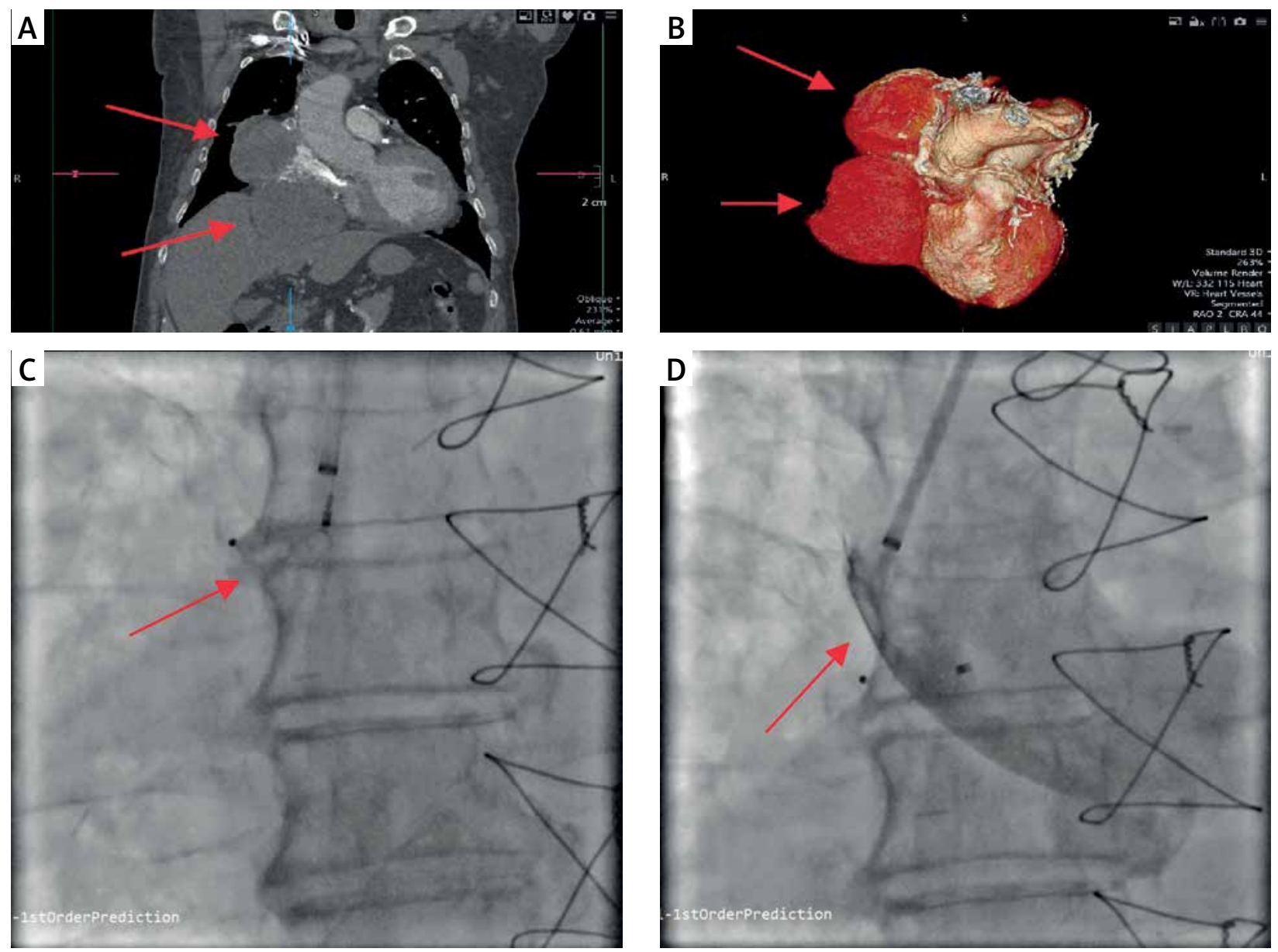

Figure 1. A, B - Computed tomography visualization of aneurysms, C - deployment of the occluder, D - aortography showing no flow inside the aneurysm

\section{References}

1. Ramirez FD, Hibbert B, Simard T, et al. Natural history and management of aortocoronary saphenous vein graft aneurysms: a systematic review of published cases. Circulation 2012; 126: 2248-56.

2. Agostoni P. How would I treat? The invited expert's opinion. Eurointervention 2014; 9: 1246.

3. Sura AC, Douglas JS. Percutaneous closure of giant saphenous vein graft aneurysm. JACC Cardiovasc Interv 2010; 3: 784-5. 